\title{
Dermato-Epidemiologie - es gibt noch viel zu tun!
}

\author{
T. Schäfer, München
}

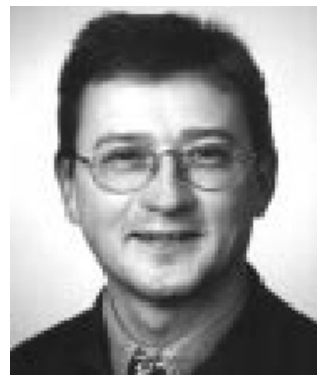

Zum zweiten Mal finden Sie im

ALLERGO JOURNAL Beiträge unserer DermatoEpidemiologie-Tagung. Dabei mag der Begriff ,Epidemiologie" beim Leser spontan nur Langeweile erzeugen, erwartet er doch allerhöchstens eine Auflistung von Häufigkeitsangaben zu Symptomen oder Erkrankungen.

Mittlerweile ist aus der Epidemiologie jedoch eine Wissenschaft geworden, die uns weit mehr als nur Frequenzangaben liefern kann. Aber selbst in diesem Bereich liegen noch große Defizite: Für Deutschland gibt es z.B. keine aussagekräftigen bevölkerungsbezogenen Daten zur Häufigkeit der Kontakt- oder Nahrungsmittelallergie - ganz abgesehen von der Frage, ob Allergien auch in Deutschland zugenommen haben. Hierzu gibt es bislang nur Berichte aus dem europäischen Ausland.

Wichtige Beiträge für präventionsmedizinische Ansätze

Epidemiologie kann aber weit mehr als nur zählen. Im sorgfältigen Vergleich von Erkrankten und Gesunden können beispielsweise Einflußfaktoren identifiziert werden, die, sofern sie gewissen Kriterien der Kausalität genügen und sich statistisch als stabil erweisen, als pathogenetisch bedeutsame Risikofaktoren in Betracht kommen.

Damit kann ein wesentlicher Beitrag zum Verständnis der Erkrankung und für präventionsmedizinische Ansätze geleistet werden. Und wenn wir heute davon ausgehen, daß Haustierhaltung oder Passivrauchen als Risikofaktoren für Allergien gelten, so geht das auch auf epidemiologische Studien zurück, die wiederholt zeigen konnten, daß diese Parameter beim Menschen und unter seinen aktuellen Lebensbedingungen Bedeutung haben. Viele Fragen sind natürlich noch offen. Wir wissen weder, warum jemand allergisch wird, noch warum Allergien zugenommen haben.

\section{Basisdaten für die wissensbasierte Medizin}

Epidemiologie aber mit Statistik gleichzusetzen, hieße das Fach unzulässig zu verkürzen. Das Gebiet reicht von der Studienplanung über die Auswahl des richtigen Studiendesigns und geeigneter Erhebungsinstrumente bis hin zu den Konzepten von systematischen und Zufallsfehlern und zur adäquaten Analyse.

Die angewandten Methoden haben dabei nicht nur für bevölkerungsbezogene Studien Relevanz, sondern auch für den wissenschaftlich Tätigen in der Planung und Durchführung experimenteller oder klinischer Studien und für jeden Leser von Fachzeitschriften, der Studienergebnisse kritisch bewerten will. Das Ergebnis der kritischen Bewertung relevanter klinischer Fachliteratur stellt wiederum die Grundlage für die sogenannte wissensbasierte Medizin (evidence based medicine) dar.

Eine formale Ausbildung in Epidemiologie wird am ehesten durch die in angelsächsischen Ländern üblichen „Master“-Abschlüsse wie Master of Public Health (MPH) oder Master of Science in Epidemiology (MSc) gewährleistet. Bislang steht die in Deutschland angebotene Ausbildung diesen noch etwas nach.
Einerseits liegt dies an der deutlich längeren Tradition des Fachs im Ausland. Während manche PublicHealth-Hochschule in den USA ihr 75jähriges Bestehen gefeiert hat, gibt es diese Ausbildung hierzulande erst seit wenigen Jahren. Zum anderen hat Public-Health-Forschung in den USA einen deutlich höheren Stellenwert in der öffentlichen Wahrnehmung.

In Deutschland gibt es bisher kaum eine Handvoll Dermatologen mit einer formalen Ausbildung in Epidemiologie. Zwei davon (Dr. B. Rzany,

Mannheim und der Autor) haben eine Arbeitsgruppe gegründet, deren vordringliches Ziel in methodischen Arbeiten auf dem Gebiet der Dermato- und Allergo-Epidemiologie besteht. Damit hoffen wir, die Qualität

epidemiologischer Forschung auf diesem Gebiet weiter zu erhöhen.

\section{Das Interesse wächst - packen wir's an}

Die folgenden Beiträge geben Ihnen einen Überblick über die Projekte, die aktuell bearbeitet werden. Für die Zukunft wünschen wir uns, daß das erwachte Interesse an dem Fach weiter wächst und daß die Bedeutung epidemiologischer Forschung im Bereich Dermatologie und Allergologie stärker in den Hochschulbereich hineinwirkt.

$\mathrm{Zu}$ tun gibt es genug - packen wir's an.

\section{Korrespondenzadresse:}

Dr. med. T. Schäfer, MPH, Klinik und Poliklinik für Dermatologie und Allergologie am Biederstein der TU München,

Biedersteiner Straße 29, D-80802 München, tschaefer@1rz.tu-muenchen.de 